\title{
Risk Combinations in the Risk Analysis and Management
}

\author{
Milík Tichý \\ Department of Civil Engineering, Czech Technical University \\ 16629 Prague, Czech Republic \\ E-mail:milik.tichy@volny.cz
}

Received 29 November 2016

Accepted 25 April 2017

\begin{abstract}
The analogies between the risks carried by entities and the physical loads affecting building and engineering structures can be applied in the studies of risks forming groups arranged according to specified definitions. Four classes of relations among risks belonging to a particular hazard are to be distinguished: existential, sequential, physical, and statistical. The type and number of possible groupings of combined risks, called "risk combinations", depends upon the type of existential and sequential relations among the risks affecting the respective entity. The results of the analysis from this study can be used in decision makings on which risks are to be considered in the decisions. The combined-risk problem relates to various fields of risk engineering and management as, such as seismic engineering, medicine, agriculture, economic engineering.
\end{abstract}

Keywords: Combined risks, multiple perils, combination, existential relation, sequential relation, estimate

\section{Introduction}

It was demonstrated by Tichý (2014) that simple analogies exist between loads affecting building and engineering structures, and hazards and risks affecting entities of various kinds.

Although physical loads on structures are tangible phenomena, and risks to entities are intangible, research methods used in the structural reliability theory can be applied in the domain of risk engineering and management.

In structural engineering, one of the main issues of the reliability theory is the problem of load combinations. The problem of combined risks, which is typical for many risk management situations, is of analogous importance, since information on combined risks is commonly needed in many situations. However, it is frequently underrated and misunderstood, or entirely ignored. One of the domains where it is traditionally paid systematic attention to is that of insurance industry where the multiple peril policies are an everyday issue, and discounts of premium rates in multiple peril poli- cies, i.e., at combined risks, is common practice. The problem of combined risks draws also the attention of authorities and governing bodies, so that rules of dealing with them become legal status, which can be seen in Multiple Peril Insurance Act of 2009, 2010 in USA, and analogous public documents.

The aim of this paper is to show that the problem of combined risks can be approached in a way similar to that developed for tangible phenomena entering the structural reliability requirements, as presented by Tichý (1994).

\section{Concepts}

\subsection{Notions and assumptions}

In this paper, the risk will be simply understood as a time and space dependent intangible phenomenon described by a quantity acquiring numerical values. The structure of such quantity can be probabilistic (Kaplan and Garrick, 1981), or non-probabilistic (Viscusi et al., 2003), or mixed. Whatever quantitative description of 
risk is applied, the numerical risk always is either a straight expert estimate, or a derived estimate, based on physical, sociological, zoological, etc., observations, or on expert judgments.

To emphasize the estimate character of the numerical risk the roman font symbol Rs instead of standard mathematical notation in italics is used. It frequently happens that Rs is calculated by sophisticated models making the impression that an exact value of risk is being obtained. Yet, such models are estimates by themselves, and so the value of Rs found is still an estimate.

Further, in this paper the following terms are applied:

- entity - covers universally all objects and processes of any kind - material, non-material, or mixed; the term also includes the entity's ambiance

- defined risk - risk that is fully described as a result of risk analysis

- undefined risk - risk that is only suspected and not sufficiently described so that the possible final loss, caused by its materialization, is unknown

- significant risk - defined risk that the decision maker considers in the decisions

- insignificant risk - risk that the decision maker assumes to be ignorable

- combined risks - all significant risks identified as affecting the entity; the concept of combined risks appears under different words and phrases: multiple risks, compound risk, multiple perils, combined hazards, etc., or it is hidden under multiple source of hazards and analogous concepts

- risk combination is a selected set of risks arranged according to specific rules; a risk combination is an intangible random phenomenon

Downside risks are presumed only; repeated risks, as well as hazard durations, and other time parameters of risks are not discussed.

The randomness of phenomena entering risk analyses is not taken into account; all concepts and variables are considered non-random, i.e., either deterministic or decision-based. It must be kept in mind that, in general, decisions are also random, though some decision makers might not be happy with such a statement. The author is well aware that the problem of combined risks cannot be fully solved without considering randomness of risk-generating phenomena.

\subsection{Combined-risks sets}

Any entity is exposed to hazards that generate a general set of risks, $\{\boldsymbol{G R} \boldsymbol{R}\}$. It consists of two distinct subsets, viz., that of undefined risks, $\{\boldsymbol{u n R s}\}$ and that of defined risks, $\{\boldsymbol{d} \boldsymbol{f} \boldsymbol{R} \boldsymbol{s}\}$. Only the latter subset will be considered. The undefined risks subset is left to be studied by the methods of the precautionary principle or by other decision-making approaches.

Assume that the risk analysis has been performed, and the set of defined risks has been identified. The decision maker now has to make many decisions on risk treatment, and, among others, he/she shall answer two questions:

- Which of the risks reported by the risk engineer/manager shall be considered significant?

The answer is entirely at the decision maker's responsibility. The decision maker has to take into account all features and circumstances of the entity. A simple arranging the risks by their numerical values would be misleading, since in certain cases, due to the severity of hazards and hazard scenarios, numerically very small risks might be of extreme importance to the entity. The selection of insignificant risks, shall be governed by social, economic, ecologic, and many others aspects.

- Which are the risk combinations that should be considered in the decisions?

This question is qualitatively different from the preceding one. The answer shall be mainly based on logical considerations.

In the following, it will be assumed that the significance question has been answered for all defined risks affecting the entity. The result is an intangible set $\{\boldsymbol{R} \boldsymbol{s}\}$ that can be both-side censored, i.e., it may not contain risks insignificant according to the decision maker's priorities, or risks transferred to other entities (insurance companies, banks, bonding societies).

One of the problems closely connected to that of combined risks, is that of the comprehensive risk, $\mathbf{R s}_{\mathrm{cmp}}$, the value of which is often required by decision makers or other persons. This paper is not dealing with the problem, as it cannot be satisfactorily solved without taking into account the randomness of risks. A simple adding up the values of all risks $\boldsymbol{R s} \in\{\boldsymbol{R} \boldsymbol{s}\}$ would be nonsensical. 


\subsection{Relations between risks}

The risks are not loosely floating in the entity's risk space. Four relation classes between the risks belonging to $\{\boldsymbol{R} \boldsymbol{s}\}$, based on intrinsic properties of the risks involved, can be distinguished:

- existential relations

- sequential relations

- physical relations

- statistical relations

However, only the first two relation classes will be discussed in the following. Simultaneously, it will be assumed that the risks belonging to $\{\boldsymbol{R} \boldsymbol{s}\}$ are mutually physically and statistically independent; i.e., no risk is considered here to be a physical or statistical function of other risks.

Classifications based on subjective properties of the risk assessor are possible, as, e.g., the classification by risk attitudes (Weber et al., 2002). Such classifications are not considered in this paper.

\section{Existential Relations}

\subsection{Four basic existential relations}

Assume that the set $\{\boldsymbol{R} \boldsymbol{s}\}$ containing all significant risks affecting the entity at a certain location and at a certain point in time, identified by risk engineers and risk managers. Let the set be analyzed from the viewpoint of simultaneous existence of significant risks. The following types of existential relations can be found between risks belonging to $\{\boldsymbol{R s}\}$ :

(a) existentially simultaneous risks cannot exist alone, as their simultaneous existence is indispensable

(b) existentially independent risks may or may not exist simultaneously

(c) existentially positively dependent risks consist of primary risks, Rs*, and of secondary risks, Rs**; risks Rs* and Rs** may or may not exist, but a risk Rs** may only exist when a risk Rs* does exist

(d) existentially negatively dependent risks never exist simultaneously; such risks exist only individually

The above phrase "may or may not exist" does not refer to the randomness of the existence of risks; the phrase is of deterministic or decision-based character. The randomness is still not anticipated here, and so the risk relations are discussed, as mentioned above, without any reference to their random nature.
The importance of existential relations appears in the determination in what existential combinations $\left(\mathrm{Rs}_{i}\right.$, $\left.\mathrm{Rs}_{k}, \ldots, \mathrm{Rs}_{l}\right)_{e}$ the combined risks can be materialized, $\mathrm{Rs}_{i}, \mathrm{Rs}_{k}, \ldots, \mathrm{Rs}_{l}$ being some of the risks $\mathrm{Rs}_{1}$ through $\mathrm{Rs}_{n}$ belonging to $\{\boldsymbol{R} \boldsymbol{s}\}$.

For completeness' sake, the existence of a single risk is regarded as a combination, supposing of course that this risk belongs to $\{\boldsymbol{R s}\}$.

\subsection{Relation symbols}

For various relations, simple symbols are used in the following:
$:\langle\bullet\rangle$
$\langle\bullet\rangle$
simultaneity, inevitability independence, possibility
primary risks, Rs*)
$\mathbf{N}\langle\bullet \quad$ negative dependence, impossibility

The bullets, $\bullet$, stand either for sets of risks, i.e., single risks and subsets of risks, or also for comprehensive relation symbols. Substituting for bullets, existential relation formulas are obtained.

In the following, the significance of the relations between risks can be simply demonstrated by examples of two risks and three risks. It should be possible to extend the discussion to larger risk sets; that would result in unnecessarily complicated elaborations without substantial merit to this paper.

\subsection{Two risks}

Assume that the Entity is exposed to two risks, $\mathrm{Rs}_{1}$ and $\mathrm{Rs}_{2}$. Consider the above described four types of existential relations among these risks:

(1) Two existentially simultaneous risks (relation formula: ! $\left.\left\langle\mathbf{R s}_{\mathbf{1}}, \mathbf{R s}_{2}\right\rangle\right)$ have only one possibility of existence, i.e., they can come out in only a single existential combination:

$$
\text { - } \mathrm{Rs}_{1} \text { and } \mathrm{Rs}_{2} \text { simultaneously: } \quad\left(\mathrm{Rs}_{1}, \mathrm{Rs}_{2}\right)_{e}
$$

A solitary existence of any of these risks is ruled out.

(2) Two existentially independent risks (relation formula: $\left\langle\mathbf{R s}_{\mathbf{1}}, \mathbf{R s}_{\mathbf{2}}\right\rangle$ ) - three existential combinations are possible:

- $\mathrm{Rs}_{1}$ alone:

$\left(\mathrm{Rs}_{1}\right)_{e}$

- $\mathrm{Rs}_{2}$ alone:

$\left(\mathrm{Rs}_{2}\right)_{e}$

- $\mathrm{Rs}_{1}$ and $\mathrm{Rs}_{2}$ simultaneously:

$\left(\mathrm{Rs}_{1}, \mathrm{Rs}_{2}\right)_{e}$ 
(3) Let $\mathrm{Rs}_{1}$ be the primary risk, $\mathrm{Rs}^{*}$, and $\mathrm{Rs}_{2}$ be the secondary risk, Rs**, existentially independent of $\mathrm{Rs}_{1}$ (relation formula: $\left.\left\langle\mathbf{R s}_{\mathbf{2}}\left[\mathbf{R s}_{\mathbf{1}}\right]\right\rangle\right)$ - only two existential combinations are possible:

- $\mathrm{Rs}_{1}$ alone:

$\left(\mathrm{Rs}_{1}\right)_{e}$

- $\mathrm{Rs}_{1}$ and $\mathrm{Rs}_{2}$ simultaneously:

$\left(\mathrm{Rs}_{1}, \mathrm{Rs}_{2}\right)_{e}$

A solitary existence of the secondary risk, $\mathrm{Rs}^{* *} \equiv$ $\mathrm{Rs}_{2}$, is impossible.
(4) Two existentially negatively dependent risks (relation formula: $\left.\mathbf{N}\left\langle\mathbf{R s}_{\mathbf{1}}, \mathbf{R s}_{\mathbf{2}}\right\rangle\right)$ - two existential combinations are possible:

- $\mathrm{Rs}_{1}$ alone:

$\left(\mathrm{Rs}_{1}\right)_{e}$

- $\mathrm{Rs}_{2}$ alone:

$\left(\mathrm{Rs}_{2}\right)_{e}$

A simultaneous existence of the two risks is impossible.

The possible existential combinations of two risks, $\mathrm{Rs}_{1}$ and $\mathrm{Rs}_{2}$, are summarized in Table 1.

Table 1. Existential combinations of two risks

( $\mathrm{X}$ - possibility, 0 - impossibility of the respective combination).

\begin{tabular}{|c|c|c|c|c|}
\hline \multirow{3}{*}{$\begin{array}{c}\text { Existential } \\
\text { combination }\end{array}$} & \multicolumn{4}{|c|}{ Case No. / Relation formula } \\
\hline & (1) & (2) & (3) & (4) \\
\hline & $!\left\langle\mathbf{R s}_{1}, \mathbf{R s}_{2}\right\rangle$ & $\left\langle\mathbf{R s}_{1}, \mathbf{R s}_{2}\right\rangle$ & $\left\langle\mathbf{R s}_{2}\left[\mathbf{R s}_{1}\right]\right\rangle$ & $\mathbf{N}\left\langle\mathbf{R s}_{1}, \mathbf{R s}_{2},\right\rangle$ \\
\hline 1: $\left(\mathrm{RS}_{1}\right)_{e}$ & 0 & $\mathrm{X}$ & $\mathrm{X}$ & $\mathrm{X}$ \\
\hline 2: $\left(\mathrm{RS}_{2}\right)_{e}$ & 0 & $\mathrm{X}$ & 0 & $\mathrm{X}$ \\
\hline 3: $\left(\mathrm{Rs}_{1}, \mathrm{Rs}_{2}\right)_{e}$ & $\mathrm{X}$ & $\mathrm{X}$ & $\mathrm{X}$ & 0 \\
\hline $\begin{array}{l}\text { Number of exis- } \\
\text { tential combina- } \\
\text { tions }\end{array}$ & 1 & 3 & 2 & 2 \\
\hline
\end{tabular}

Table 2a. Existential combinations of three risks at some arrangements of the existential relations (X - possibility, 0 - impossibility of the respective combination).

\begin{tabular}{|c|c|c|c|c|}
\hline \multirow{3}{*}{$\begin{array}{l}\text { Existential } \\
\text { combination }\end{array}$} & \multicolumn{4}{|c|}{ Case No. / Relation formula } \\
\hline & (5) & (6) & (7) & (8) \\
\hline & $!\left\langle\mathbf{R s}_{\mathbf{1}}, \mathbf{R s}_{\mathbf{2}}, \mathbf{R s}_{3}\right\rangle$ & $\left\langle\mathbf{R s}_{\mathbf{1}}, \mathbf{R s}_{2}, \mathbf{R s}_{3}\right\rangle$ & $\left\langle\operatorname{Rs}_{3}\left[\operatorname{Rs}_{2}\left[\operatorname{Rs}_{1}\right]\right]\right\rangle$ & $\begin{array}{r}\left\langle\mathbf{R s}_{3}\left[\mathbf{R s}_{2}\right],\right. \\
\left.\mathbf{N}\left\langle\mathbf{R s}_{1}, \mathbf{R s}_{2}\right\rangle\right\rangle\end{array}$ \\
\hline 1: $\quad\left(\mathrm{Rs}_{1}\right)_{e}$ & 0 & $\mathrm{X}$ & $\mathrm{X}$ & $\mathrm{X}$ \\
\hline 2: $\left(\mathrm{Rs}_{2}\right)_{e}$ & 0 & $\mathrm{X}$ & 0 & $\mathrm{X}$ \\
\hline 3: $\quad\left(\mathrm{Rs}_{3}\right)_{e}$ & 0 & $\mathrm{X}$ & 0 & 0 \\
\hline 4: $\quad\left(\mathrm{Rs}_{1}, \mathrm{Rs}_{2}\right)_{e}$ & 0 & $\mathrm{X}$ & $\mathrm{X}$ & 0 \\
\hline 5: $\quad\left(\mathrm{Rs}_{1}, \mathrm{Rs}_{3}\right)_{e}$ & 0 & $\mathrm{X}$ & 0 & $\mathrm{X}$ \\
\hline 6: $\quad\left(\mathrm{Rs}_{2}, \mathrm{Rs}_{3}\right)_{e}$ & 0 & $\mathrm{X}$ & 0 & 0 \\
\hline 7: $\quad\left(\mathrm{Rs}_{1}, \mathrm{Rs}_{2}, \mathrm{Rs}_{3}\right)_{e}$ & $\mathrm{X}$ & $\mathrm{X}$ & $\mathrm{X}$ & 0 \\
\hline $\begin{array}{l}\text { Number of } \\
\text { existential } \\
\text { combinations }\end{array}$ & 1 & 7 & 3 & 3 \\
\hline
\end{tabular}


Table $2 \mathrm{~b}$. Existential combinations of three risks with some arrangements of existential relations (X - possibility, 0 - impossibility of the respective combination).

\begin{tabular}{|c|c|c|c|c|}
\hline & \multirow{3}{*}{$\begin{array}{l}\text { Existential } \\
\text { combination }\end{array}$} & \multicolumn{3}{|c|}{ Case No. / Relation formula } \\
\hline & & (9) & (10) & (11) \\
\hline & & $\left\langle\mathbf{R s}_{3},\left\lfloor\left\langle\mathbf{R s}_{1}, \mathbf{R s}_{2}\right\rangle\right\rangle\right.$ & $\mathbf{N}\left\langle\mathbf{R s}_{3}, \mathbf{R s}_{1}, \mathbf{R s}_{2}\right\rangle$ & $\left\langle\mathbf{R s}_{3},\left[!\left\langle\mathbf{R s}_{1}, \mathbf{R s}_{2}\right\rangle\right]\right\rangle$ \\
\hline 1: & $\left(\mathrm{Rs}_{1}\right)_{e}$ & 0 & $\mathrm{X}$ & $\mathrm{X}$ \\
\hline 2: & $\left(\mathrm{Rs}_{2}\right)_{e}$ & 0 & $\mathrm{x}$ & $\mathrm{X}$ \\
\hline 3: & $\left(\mathrm{Rs}_{3}\right)_{e}$ & $\mathrm{X}$ & $\mathrm{x}$ & 0 \\
\hline 4: & $\left(\mathrm{Rs}_{1}, \mathrm{Rs}_{2}\right)_{e}$ & $\mathrm{X}$ & $\mathrm{X}$ & 0 \\
\hline 5: & $\left(\mathrm{Rs}_{1}, \mathrm{Rs}_{3}\right)_{e}$ & 0 & $\mathrm{x}$ & $\mathrm{X}$ \\
\hline 6: & $\left(\mathrm{Rs}_{2}, \mathrm{Rs}_{3}\right)_{e}$ & 0 & $\mathrm{x}$ & $\mathrm{X}$ \\
\hline & $\left(\mathrm{Rs}_{1}, \mathrm{Rs}_{2}, \mathrm{Rs}_{3}\right)_{e}$ & $\mathrm{X}$ & 0 & 0 \\
\hline & $\begin{array}{l}\text { mber of existential } \\
\text { nbinations }\end{array}$ & 3 & 6 & 4 \\
\hline
\end{tabular}

\subsection{Three risks}

Whereas only four possible relations can be found for two risks [these relations are identical with the above formulated existential relations (a) through (d)], a much larger set of combination possibilities is offered by three risks, $\mathrm{Rs}_{1}, \mathrm{Rs}_{2}$, and $\mathrm{Rs}_{3}$. Let us introduce here only some cases:

(5) All three risks are existentially simultaneous (relation formula: ! $\left.\left\langle\mathbf{R s}_{\mathbf{1}}, \mathbf{R s}_{\mathbf{2}}, \mathbf{R s}_{\mathbf{3}}\right\rangle\right)$ - only one existential combination can exist:

$$
\left(\mathrm{Rs}_{1}, \mathrm{Rs}_{2}, \mathrm{Rs}_{3}\right)_{e}
$$

(6) All three risks are existentially independent (relation formula: $\left\langle\mathbf{R s}_{\mathbf{1}}, \mathbf{R s}_{\mathbf{2}}, \mathbf{R s}_{\mathbf{3}}\right\rangle$ ) - seven combinations are possible (cf. Table 2a):

$$
\begin{array}{lll}
\left(\mathrm{Rs}_{1}\right)_{e} & \left(\mathrm{Rs}_{1} \mathrm{Rs}_{2}\right)_{e} & \left(\mathrm{Rs}_{1}, \mathrm{Rs}_{2}, \mathrm{Rs}_{3}\right)_{e} \\
\left(\mathrm{Rs}_{2}\right)_{e} & \left(\mathrm{Rs}_{1} ; \mathrm{Rs}_{3}\right)_{e} \\
\left(\mathrm{Rs}_{3}\right)_{e} & \left(\mathrm{Rs}_{2}, \mathrm{Rs}_{3}\right)_{e}
\end{array}
$$

(7) Let $\mathrm{Rs}_{2}$ be positively dependent on $\mathrm{Rs}_{1}$, and $\mathrm{Rs}_{3}$ positively dependent on $\mathrm{Rs}_{2}$ (relation formula: $\left\langle\mathbf{R s}_{\mathbf{3}}\right.$ $\left.\left.\left[\mathbf{R s}_{\mathbf{2}}\left[\mathbf{R s}_{\mathbf{1}}\right]\right]\right\rangle\right)$. The existential combinations possible in this case are shown in Table $2 \mathrm{a}$.

(8) Let risks $\mathrm{Rs}_{1}$ and $\mathrm{Rs}_{2}$ be negatively dependent, and let $\mathrm{Rs}_{3}$ depend positively on $\mathrm{Rs}_{1}$ (i.e., the relation formula is: $\left.\left\langle\mathbf{R s}_{\mathbf{3}}\left[\mathbf{R} \mathbf{s}_{\mathbf{1}}\right], \mathbf{N}\left\langle\mathbf{R s}_{\mathbf{1}}, \mathbf{R s}_{\mathbf{2}}\right\rangle\right\rangle\right)$.

Possible existential combinations are shown in Table 2a.
(9) Let $\mathrm{Rs}_{1}$ and $\mathrm{Rs}_{2}$ be existentially simultaneous, with $\mathrm{Rs}_{3}$ existentially independent of $\mathrm{Rs}_{1}$ and $\mathrm{Rs}_{2}$ (relation formula $\left.\left\langle\mathbf{R s}_{\mathbf{3}}, !\left\langle\mathbf{R s}_{\mathbf{1}} \mathbf{R s}_{\mathbf{2}}\right\rangle\right\rangle\right)$ - Possible existential combinations are shown in Table $2 \mathrm{~b}$.

(10) The three risks cannot exist simultaneously (relation formula: $\mathbf{N}\left\langle\mathbf{R s}_{\mathbf{1}}, \mathbf{R s}_{\mathbf{2}}, \mathbf{R s}_{\mathbf{3}}\right\rangle$ ) - see Table $2 \mathrm{~b}$.

(11) Let $\mathrm{Rs}_{3}$ be existentially dependent either on $\mathrm{Rs}_{1}$, or on $\mathrm{Rs}_{2}$, but $\mathrm{Rs}_{1}$ and $\mathrm{Rs}_{2}$ be mutually exclusive (relation formula: $\left.\left\langle\mathbf{R s}_{\mathbf{3}}\left[\mathbf{N}\left\langle\mathbf{R s}_{\mathbf{1}}, \mathbf{R s}_{\mathbf{2}}\right)\right]\right\rangle\right)$ - see Table 2 b.

Observe that the nature of existential combinations differs according to the type of the relation formula. When considering combinations of two or more risks, three types of existential combinations can be distinguished (see Tables 1 through 3):

- closed combinations, where all risks are existentially simultaneous [e.g., combination No. 3 in case (1), combination No. 7 in case (5), and combination No. 4 in case (9)];

- fixed combinations, where at least one risk is existentially independent of the others, and at least one risk is primary [e.g., combination No. 3 in case (3), combinations Nos. 4 and 7 in case (7), combination No. 5 in case (8), combination No. 7 in case (9)];

- open combinations, where none of the risks is bound to other risks [e.g., combination No. 3 in case (2), combinations Nos. 4 through 7 in case (6)]. 


\subsection{Number of existential combinations}

The foregoing paragraphs show that the number of existential combinations, in which risks can exist, depends on the type of the respective existential relations.

When existentially simultaneous risks are dealt with, just one existential combination is possible; i.e.,

$$
m_{e}=1
$$

For a set $\{\boldsymbol{R s}\}$ of existentially independent risks, $\mathrm{Rs}_{1}, \mathrm{Rs}_{2}, \ldots, \mathrm{Rs}_{n}$, the number $m_{e}$ of possible existential combinations of $n$ risks is expressed by

$$
m_{e}=\sum_{k=1}^{n}\left(\begin{array}{l}
n \\
k
\end{array}\right)
$$

where $k=$ number of risks in the respective combination.-Note that non-ordered combinations are dealt with as the order of combined risks is of no importance.

The number of existential combinations referred to a set $\{$ Rs $\}$ of $n$ risks that are either positively or negatively existentially dependent is given by

$$
m_{e}=\sum_{k=1}^{n}\left(\begin{array}{l}
n \\
k
\end{array}\right)-\bar{m}_{e}
$$

where $\bar{m}_{e}=$ number of existential combinations that cannot exist. A general formula for $\bar{m}_{e}$ can not be presented, since the diversity of relations is unlimited. Moreover, such a formula would be without practical significance. It suffices to find $\bar{m}_{e}$ by simple judgment.

When in a set of $n$ risks a subset consisting of $r$ simultaneous risks is present, and, at the same time the remaining $(n-r)$ risks are existentially independent, the subset of simultaneous risks should be considered as a single risk. Thus, the number of existential combinations is:

$$
m_{e}=\sum_{k=1}^{n-r+1}\left(\begin{array}{c}
n-r+1 \\
k
\end{array}\right)
$$

\section{Sequential Relations}

\subsection{Seven basic sequential relations}

Decision makers in many sectors rarely understand that a risk can generate further risks; consequently, they do not take this fact into account. Scores of example can be given.
While the existential combinations concern risks that affect an entity at a specific moment, $t$, throughout the reference period $T_{\text {ref, }}$, the sequential relations refer to entities that might be exposed to risks physically and statistically independent along the time line.

Consider a set $\{\boldsymbol{R} \boldsymbol{s}(\boldsymbol{t})\}$ of time-dependent risks, $\mathrm{Rs}_{1}(t), \mathrm{Rs}_{2}(t), \ldots, \mathrm{Rs}_{n}(t)$, taking into attention possibilities of their successive existence. Here, time dependence of risk $\mathrm{Rs}_{i}(t)$ means that the particular risk can occur along the timeline in $T_{\text {ref }}$. The possibility of repeated incidence of $\mathrm{Rs}_{i}(t)$ will not be considered here; yet, it cannot be excluded.

Between the individual risks in $T_{\text {ref }}$, the following types of sequential relations can be distinguished:

(a) sequentially inevitable non-ordered risks all follow one after the other in an arbitrary order

(b) sequentially free non-ordered risks may follow in an arbitrary order, and some of them may not take place

(c) sequentially inevitable ordered risks all follow in a specified order

(d) sequentially free ordered risks follow in a specified order, but some may not come up

(e) sequentially excluding risks can not follow one after the other

(f) sequentially backwards dependent risks are formed by a subset of primary risks, $\operatorname{Rs}(t)^{*}$, and by a subset of secondary risks, $\operatorname{Rs}(t)^{* *}$; risks $\operatorname{Rs}(t)^{* *}$ can only appear when they are preceded by risks $\operatorname{Rs}(t)^{*}$; any subset can consist of one or more elements

(g) sequentially forwards dependent risks are analogous to the foregoing type: $\operatorname{Rs}(t)^{* *}$ can only come out if they are followed by $\operatorname{Rs}(t)^{*}$

Similarly as in the case of existential relations, sequential relations determine which sequential combinations are possible in a particular case. A sequential combination is defined by successive existence of several risks belonging to the set $\{\boldsymbol{R s}(\boldsymbol{t})\}$. Notation

$$
\left[\operatorname{Rs}_{i}(t), \operatorname{Rs}_{k}(t), \ldots, \operatorname{Rs}_{l}(t)\right]_{s}
$$

will be used for sequential combinations, where $i, k, l$ $=$ subscripts referring to the time-dependent risks that belong to the set $\{\boldsymbol{R} \boldsymbol{s}(\boldsymbol{t})\}$.

For simplicity, the time argument, $(t)$, is omitted in the subsequent paragraphs. 


\subsection{Relation symbols}

Again, relation formulas can be written to describe sequential combinations. The following symbols will be used:

$!(\bullet\rangle \quad$ certainty, inevitability

$\langle\bullet\rangle \quad$ independence, possibility

$\mathbf{N}\langle\bullet\rangle$ negative dependence, impossibility

- $\| \cdot$ orderliness

$\bullet \sim \bullet$ non-orderliness

$\bullet \leftarrow$ backwards dependence

$\bullet \rightarrow \bullet$ forwards dependence

As before, bullets stand either for sets of risks, i.e., single risks and groups of risks, or also for a lower relation symbol. Substituting for bullets sequential relation formulas are obtained.

\subsection{Three risks}

The individual sequential relations and the way of their presentation can be demonstrated by some cases of three risks forming the set $\{\boldsymbol{R s}\}$ :

(a) Three sequentially non-ordered unavoidable risks, i.e., the three risks must follow each other in an arbitrary order (relation formula: ! $\left.\left\langle\mathbf{R s}_{\mathbf{1}} \sim \mathbf{R s}_{\mathbf{2}} \sim \mathbf{R s}_{3}\right\rangle\right)$ six sequential combinations exist:

$$
\begin{array}{ll}
\left(\mathrm{Rs}_{1}, \mathrm{Rs}_{2}, \mathrm{Rs}_{3}\right)_{s} & \left(\mathrm{Rs}_{2}, \mathrm{Rs}_{3}, \mathrm{Rs}_{1}\right)_{s} \\
\left(\mathrm{Rs}_{1}, \mathrm{Rs}_{3}, \mathrm{Rs}_{2}\right)_{s} & \left(\mathrm{Rs}_{3}, \mathrm{Rs}_{1}, \mathrm{Rs}_{2}\right)_{s} \\
\left(\mathrm{Rs}_{2}, \mathrm{Rs}_{1}, \mathrm{Rs}_{3}\right)_{s} & \left(\mathrm{Rs}_{3}, \mathrm{Rs}_{2}, \mathrm{Rs}_{1}\right)_{s}
\end{array}
$$

Obviously, in terms of combinatory the above combinations are permutations of $\mathrm{Rs}_{i}$.

(b) For three sequentially non-ordered possible risks (relation formula $\left\langle\mathbf{R s}_{\mathbf{1}} \sim \mathbf{R s}_{\mathbf{2}} \sim \mathbf{R s}_{\mathbf{3}}\right\rangle-15$ possibilities of successive incidence can be found:

$$
\begin{array}{lll}
\left(\mathrm{Rs}_{1}\right)_{s} & \left(\mathrm{Rs}_{1}, \mathrm{Rs}_{2}\right)_{s} & \left(\mathrm{Rs}_{1}, \mathrm{Rs}_{2}, \mathrm{Rs}_{3}\right)_{s} \\
\left(\mathrm{Rs}_{2}\right)_{s} & \left(\mathrm{Rs}_{1}, \mathrm{Rs}_{3}\right)_{s} & \left(\mathrm{Rs}_{1}, \mathrm{Rs}_{2}, \mathrm{Rs}_{3}\right)_{s} \\
\left(\mathrm{Rs}_{3}\right)_{s} & \left(\mathrm{Rs}_{2}, \mathrm{Rs}_{1}\right)_{s} & \left(\mathrm{Rs}_{2}, \mathrm{Rs}_{1}, \mathrm{Rs}_{3}\right)_{s} \\
- & \left(\mathrm{Rs}_{2}, \mathrm{Rs}_{3}\right)_{s} & \left(\mathrm{Rs}_{2}, \mathrm{Rs}_{3}, \mathrm{Rs}_{1}\right)_{s} \\
- & \left(\mathrm{Rs}_{3}, \mathrm{Rs}_{1}\right)_{s} & \left(\mathrm{Rs}_{3}, \mathrm{Rs}_{1}, \mathrm{Rs}_{2}\right)_{s} \\
- & \left(\mathrm{Rs}_{3}, \mathrm{Rs}_{2}\right)_{s} & \left(\mathrm{Rs}_{3}, \mathrm{Rs}_{2}, \mathrm{Rs}_{1}\right)_{s}
\end{array}
$$

(c) For sequentially ordered unavoidable risks (relation formula: ! $\left.\left\langle\mathbf{R s}_{1}\left\|\mathbf{R s}_{\mathbf{2}}\right\| \mathbf{R s}_{3}\right\rangle\right)$ - only a single sequential combination is possible:

$$
\left(\mathrm{Rs}_{1}, \mathrm{Rs}_{2}, \mathrm{Rs}_{3}\right)_{s}
$$

(d) With sequentially ordered free risks, (relation formula: $\left\langle\mathbf{R} \mathbf{s}_{\mathbf{1}}\left\|\mathbf{R s}_{\mathbf{2}}\right\| \mathbf{R s}_{\mathbf{3}}\right\rangle$ - seven sequential combinations can be identified:

$$
\begin{array}{lll}
\left(\mathrm{Rs}_{1}\right)_{s} & \left(\mathrm{Rs}_{1}, \mathrm{Rs}_{2}\right)_{s} \\
\left(\mathrm{Rs}_{2}\right)_{s} & \left(\mathrm{Rs}_{2}, \mathrm{Rs}_{3}\right)_{s} \\
\left(\mathrm{Rs}_{3}\right)_{s} & \left(\mathrm{Rs}_{1}, \mathrm{Rs}_{3}\right)_{s}
\end{array}
$$

(e) For sequentially exclusive risks (relation formula: $\left.\mathrm{N}\left\langle\mathbf{R s}_{\mathbf{1}} \sim \mathbf{R s}_{\mathbf{2}} \sim \mathbf{R s}_{\mathbf{3}}\right\rangle\right)$ - three single-risk sequential combinations can take place:

$$
\begin{aligned}
& \left(\mathrm{Rs}_{1}\right)_{s} \\
& \left(\mathrm{Rs}_{2}\right)_{s} \\
& \left(\mathrm{Rs}_{3}\right)_{s}
\end{aligned}
$$

(f) When, for example, risks are sequentially backwards dependent according to relation formula $\left\langle\mathbf{R s}_{\mathbf{1}} \rightarrow\left\langle\mathbf{R s}_{\mathbf{2}} \sim \mathbf{R s}_{3}\right\rangle\right\rangle-$ five possible combinations can occur:

$$
\begin{array}{lll}
\left(\mathrm{Rs}_{1}\right)_{\mathrm{s}} & \left(\mathrm{Rs}_{1}, \mathrm{Rs}_{2}\right)_{\mathrm{s}} & \left(\mathrm{Rs}_{1}, \mathrm{Rs}_{2}, \mathrm{Rs}_{3}\right)_{\mathrm{s}} \\
- & \left(\mathrm{Rs}_{1}, \mathrm{Rs}_{3}\right)_{\mathrm{s}} & \left(\mathrm{Rs}_{1}, \mathrm{Rs}_{3}, \mathrm{Rs}_{2}\right)_{\mathrm{s}}
\end{array}
$$

Observe that $\mathrm{Rs}_{2}$ is the primary risk, and $\left(\mathrm{Rs}_{2} \sim \mathrm{Rs}_{3}\right)$ is the secondary group.

(g) Plainly, three risks can also be in other $a$ posteriori relations, as for example

$$
\begin{aligned}
& \left\langle\mathbf{N}\left\langle\mathbf{R s}_{1} \sim \mathbf{R s}_{2}\right\rangle \rightarrow \mathbf{R s}_{3}\right\rangle \\
& \left\langle\mathbf{R s}_{1} \rightarrow !\left\langle\mathbf{R s}_{2}, \mathbf{R s}_{3}\right\rangle\right\rangle
\end{aligned}
$$

The possible sequential combinations can be easily determined.

(h) Similar conclusion is valid for a backwards dependence. For example, if

$$
\left\langle\mathbf{R s}_{1} \leftarrow !\left\langle\mathbf{R s}_{2} \leftarrow \mathbf{R s}_{3}\right\rangle\right\rangle
$$

the following two sequential combinations are possible:

$$
\left(\mathrm{Rs}_{2}, \mathrm{Rs}_{3}\right)_{s} \quad\left(\mathrm{Rs}_{1}, \mathrm{Rs}_{2}, \mathrm{Rs}_{3}\right)_{s}
$$

\subsection{Number of sequential combinations}

The previous paragraphs suggest that the number of sequential combinations depends again on the type of the particular sequential relation. However, this number is likely greater than for existential combinations. For $n$ sequentially free non-ordered risks, i.e., for $\left\langle\mathbf{R s}_{\mathbf{1}} \sim \mathbf{R s}_{\mathbf{2}} \sim\right.$ $\left.\ldots \sim \mathbf{R s}_{n}\right\rangle$, the number of sequential combinations, $m_{s}$, is mathematically given by 


$$
m_{s}=\sum_{k=1}^{n} \frac{n !}{(n-k) !}
$$

The number $m_{s}$ can be easily determined for the following sequential relations:

$$
\begin{array}{ll}
!\left\langle\mathbf{R s}_{\mathbf{1}} \sim \mathbf{R s}_{\mathbf{2}} \sim \ldots \sim \mathbf{R s}_{n}\right\rangle: & m_{s}=n ! \\
!\left\langle\mathbf{R s}_{\mathbf{1}}\left\|\mathbf{R s}_{\mathbf{2}}\right\| \ldots \| \mathbf{R s}_{\boldsymbol{n}}\right\rangle: & m_{s}=1 \\
\mathbf{N}\left\langle\mathbf{R s}_{\mathbf{1}} \sim \mathbf{R s}_{\mathbf{2}} \sim \ldots \sim \mathbf{R s}_{n}\right\rangle: & m_{s}=n
\end{array}
$$

The number of sequential combinations related to the "certainty/non-orderliness" relation formula, ! $\left\langle\mathbf{R s}_{\mathbf{1}} \sim\right.$ $\left.\mathbf{R s}_{\mathbf{2}} \sim \ldots \sim \mathbf{R s}_{n}\right\rangle$, is obviously defined by the number of permutations of the respective risks. When risks are backwards or forwards dependent, the determination of $m_{s}$ gets complicated, because also the order of risks must be respected. Fortunately, requirements for the number of combinations do not apply in practice,

\section{Conclusions}

Risk engineers or risk managers shall pay attention to the properties of the existing risks when assessing the risk exposure of an entity. In a general case, not all of the risks identified may act simultaneously: some may be absent at specific situations; some may be eliminated by other risks; some may follow in an arbitrary order, etc. However these aspects of the combined risks are often underrated and misunderstood.

In the above analysis the following conclusions have been reached:

- in a specific case of combined risks, only some of the risks identified are considered as significant by the decision maker;

- the decision maker's assessment of the risk significance is entirely in his/her hands;

- the possible patterns of the risk combinations are governed by mutual relations between significant risks;

- two basic kinds of relations between risks determine the significant risk combinations - existential relations and sequential relations;

- the number of possible existential and sequential combinations depends on the character of mutual relations between risks and groups of risks.

\section{References}

S. Kaplan and B. J. Garrick, On the quantitative definition of risk, J. of Risk Analysis, 1(1) (1981), 11-27.

Multiple Peril Insurance Act of 2009, H.R. 1264, 111th Congress (2009-2010). United States.

M. Tichý, Applied Methods of Structural Reliability (Kluwer Academic Publishers, Dordrecht 1994).

M. Tichý, Entity risk mechanics, J. of Risk Analysis and Crisis Response, 2(2) (2012),107-113.

M. Tichý, Analogies in entity risk mechanics, J. of Risk Analysis and Crisis Response, 4(2) (2014), 96-107.

W. K. Viscusi and K. K. Hakes: Risk ratings that do not measure probabilities, J. of Risk Research, 6(1) (2003), 23-43.

E. U. Weber, A.-R. Blais and N. E. Betz, A domain-specific risk-attitude scale: Measuring risk perceptions and risk behaviors, J. of Behavioral Decision Making, 15(4) (2002), 263-290. 